\title{
Advanced Bio-signal Analytics for Continuous Bedside Monitoring of Aneurysmal Subarachnoid Hemorrhage: The Future
}

Frederick A. Zeiler ${ }^{1,2,3,4,5^{*}}$ (1)

(C) 2021 Springer Science+Business Media, LLC, part of Springer Nature and Neurocritical Care Society

Over the past two decades, there has been a dramatic expansion of cerebral physiologic monitoring devices in neurocritical care [1-4]. This type of monitoring can take many forms, including, but not limited to, intracranial pressure (ICP), cerebral perfusion pressure (CPP), brain tissue oxygen $\left(\mathrm{PbtO}_{2}\right)$, near-infrared spectroscopy (NIRS) regional cerebral oxygen saturations, transcranial Doppler (TCD) cerebral blood flow velocity assessments, thermal diffusion-based cerebral blood flow (CBF), and cerebral microdialysis. Such devices have seen a large uptake in the multimodal monitoring (MMM) of cerebral physiology in adult traumatic brain injury (TBI), with support from international experts $[1,3]$, adoption within recent renditions of guideline-based therapeutic strategies [5-7], and sparking ongoing randomized control trials on therapeutic targets based on the raw data provided from such devices $[8,9]$.

Aside from the raw cerebral physiologic information provided from these data, advances in offline and bedside bio-signal analytic platforms and techniques have led to derivation of additional indices of cerebral physiologic function. Again, the majority of the literature in this area pertains to adult TBI populations, with derived measures including those related to cerebrovascular reactivity (i.e., cerebral autoregulation) $[10,11]$, cerebral compensatory

\footnotetext{
*Correspondence: frederick.zeiler@umanitoba.ca

'Section of Neurosurgery, Department of Surgery, Rady Faculty of Health

Sciences, University of Manitoba, Winnipeg, Canada

Full list of author information is available at the end of the article
}

Invited Commentary to: https://doi.org/10.1007/s12028-020-01162-4. This manuscript requires the followingcrosslink: https://doi.org/10.1007/ s12028-020-01168-y.

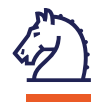

\section{Springer}

reserve [12, 13], signal complexity (i.e., entropy) [14, 15], and autonomic function [16, 17], to name a few. Cerebrovascular reactivity monitoring, taking the form of continuously updating Pearson correlation coefficients, derived from the relationship between slow-wave vasogenic fluctuations in a driving pressure for $\mathrm{CBF}$ and a surrogate measure of pulsatile cerebral blood volume/CBF, has seen increasing adoption within MMM of the TBI patient $[11,18]$. The pressure reactivity index $(\mathrm{PRx})$ is one such example, and the most commonly recognized cerebrovascular reactivity metric, derived from the relationship between ICP and mean arterial pressure (MAP) [10].

$\mathrm{PRx}$ has a strong independent association with 6-month outcome in TBI, beyond that of ICP, when adjusting for baseline admission characteristics [1923]. In addition, PRx has received some validation in experimental models as a measure of the Lassen autoregulatory curve [24-26], with defined thresholds associated with outcome in the adult TBI populations $[20,27]$. Further, recent analysis suggests that during the current era of guideline-based therapeutics in TBI, the majority of cerebral physiologic derangement is related to impaired cerebrovascular reactivity [28, 29], which remains independent to current therapeutic interventions [30]. In corollary, such cerebrovascular reactivity metrics can be used to derive other personalized physiologic targets in TBI care, with optimal CPP (CPPopt) being the exemplar [31, 32], with time spent away from CPPopt demonstrating a stronger association with outcome, compared to Brain Trauma Foundation (BTF)-based CPP thresholds [33]. This has sparked ongoing phase II studies on CPPopt vs BTF-based CPP 
targets in adult TBI [9]. Finally, dosing or exposure time to certain cerebral physiologic derangements is emerging as an increasingly important factor dictating outcome in TBI care [34-36].

Despite all of these promising advances in MMM, it is clear that the main focus has been in adult TBI populations. This leaves one uncertain as to its applicability in other neurological conditions requiring critical care management. In particular, one other patient population typically requiring invasive monitoring during their acute neurocritical care phase is the aneurysmal subarachnoid hemorrhage (aSAH) population. The main reason for the knowledge gap in advanced MMM/bio-signal analytics in aSAH patients is related to the need for proper patient volumes, high-frequency physiologic data capture, and expertise in biomedical engineering and data science. The previous literature had suggested a role for MMM and cerebrovascular reactivity monitoring in aSAH populations [37-41]. This work had documented the link between impaired PRx values and poor 6-month outcome in aSAH. Similarly, this early work had suggested the potential for continuous PRx monitoring to detect cerebral vasospasm and subsequent clinical deterioration [38]. The main limitation of the previous work has been limited patient numbers and the questionable ability to extrapolate the findings of these important initial works to other aSAH populations.

The recent publication by Svedung Wettervik et al. in Neurocrit Care [42] is a major step forward in our understanding of integrated MMM and bio-signal analytic approaches in aSAH patients, and should be considered a seminal work in the field. To date, this is the largest population of aSAH patients with high-frequency digital physiologic recordings described. In addition to this, there has been a thoughtful analysis of ICP, CPP, PRx, and CPPopt during the acute phase of their intensive care unit (ICU) stay, providing some of the first cerebral physiologic dysfunction dosing/exposure assessments. As with the TBI population [34-36], Svedung Wettervik et al. have been able to correlate time spent with ICP above $20 \mathrm{mmHg}$, CPP below various thresholds, and PRx above +0.05 (a threshold defined in TBI populations) [20], to be associated with worse outcome in aSAH. PRx was seen, as with the preliminary smaller works [37, 38], to be much more deranged in those developing symptomatic cerebral vasospasm. Further to this, they provide some of the first CPPopt derivations in aSAH patients [39], extending upon the prior aSAH and extensive TBI work in the field [33]. These findings provide validation of the utilization of MMM and derived metrics for prognostication and monitoring in aSAH, while also bolstering support of the potential role of cerebrovascular reactivity metrics for vasospasm detection/monitoring.
The analysis outlined in the work by Svedung Wettervik et al. provides a platform for moving forward in the field of advanced monitoring in aSAH. However, it must be emphasized that the results highlighted should not be taken as absolute, nor adopted for routine monitoring in aSAH populations at this time. Much further work is required, which will necessitate more complex analytic strategies and expanding to multicenter collaborative efforts. Natural starting points could focus on existing data sets, exploring dose/time burden in more detail, similar to recent works in the TBI populations, using contour analysis [34-36]. Further, establishing critical thresholds associated with outcome in aSAH is required, as has been conducted in the TBI cohort [20,27], while also exploring CPPopt in more detail, perhaps using alternative ICP-derived cerebrovascular reactivity measures, such as those derived in the TBI populations from pulse waveform analysis of ICP [43, 44].

Expanding beyond existing data sets, clearly there is need for multicenter collaboration to collect expansive larger data sets and provide further validation to the results from this current study. Aside from validation, such data collection strategies could benefit from linking high-fidelity cerebral physiologic information from MMM platforms, with protein biomarker information from cerebrospinal fluid (CSF), microdialysate, and serum. Such proteome information linked with MMM physiologic data could provide insight into cellular/molecular pathways involved in secondary insult after aSAH. Furthermore, the addition of genomic data from genome-wide association studies, and epigenomic information related to biological aging, may improve our understanding of the impact of genetic variation and biological age on cerebral physiologic response post-aSAH. Such omics approaches, integrating physiome, proteome, and genome/epigenome data, would require multi-disciplinary teams consisting of: clinicians, physiologists, biomedical engineers, biologists, geneticists, epidemiologists, and data scientists. Further, such work would necessitate the use of big data strategies, time series approaches for temporally resolved data, and application of machine learning/artificial intelligence techniques in order to understand such complex data sets.

Despite the complexity of the above potential approach, this could help us better understand pathways involved in cerebral physiologic dysfunction post-aSAH, improve prognostication models, and lead to the development of personalized therapeutic approaches, including individual physiologic thresholds and pharmacologic targets, focused on reduction/elimination of secondary brain injury. The basis of such approaches is routed in highresolution MMM and cerebral physiologic signal analytics. The work from Svedung Wettervik et al. in the aSAH 
population provides that crucial platform for us to move forward in the development of personalized medicine in aSAH care, and they should be applauded for their contribution to science.

\begin{abstract}
Author details
${ }^{1}$ Section of Neurosurgery, Department of Surgery, Rady Faculty of Health Sciences, University of Manitoba, Winnipeg, Canada. ${ }^{2}$ Department of Human Anatomy and Cell Science, Rady Faculty of Health Sciences, University of Manitoba, Winnipeg, MB, Canada. ${ }^{3}$ Biomedical Engineering, Price Faculty of Engineering, University of Manitoba, Winnipeg, Canada. ${ }^{4}$ Centre on Aging, University of Manitoba, Winnipeg, Canada. ${ }^{5}$ Division of Anaesthesia, Department of Medicine, Addenbrooke's Hospital, University of Cambridge, Cambridge, UK.
\end{abstract}

\section{Source of support}

FAZ receives research support from the Manitoba Public Insurance (MPI) Neuroscience/TBI Research Endowment, the Health Science Centre Foundation Winnipeg, the United States National Institutes of Health $(\mathrm{NIH})$ through the National Institute of Neurological Disorders and Stroke (NINDS)(Grant \#: R03NS1 14335-01), the Canadian Institutes of Health Research (CIHR)(Grant \#: 432061), the Canada Foundation for Innovation (CFI)(Project \#: 38583), Research Manitoba (Grant \#: 3906), the University of Manitoba VPRI Research Investment Fund (RIF), the University of Manitoba Centre on Aging, and the University of Manitoba Rudy Falk Clinician-Scientist Professorship.

\section{Conflict of interest}

FAZ has no conflicts or disclosures.

\section{Publisher's Note}

Springer Nature remains neutral with regard to jurisdictional claims in published maps and institutional affiliations.

Received: 18 November 2020 Accepted: 24 November 2020 Published online: 5 January 2021

\section{References}

1. Hutchinson P, O'Phelan K. Participants in the international multidisciplinary consensus conference on multimodality monitoring: cerebral metabolism. Neurocrit Care. 2014;21 (2):S148-158.

2. Hutchinson PJ, Jalloh I, Helmy A, Carpenter KLH, Rostami E, Bellander B-M, et al. Consensus statement from the 2014 International Microdialysis Forum. Intensive Care Med. 2015;41:1517-28.

3. Le Roux P, Menon DK, Citerio G, Vespa P, Bader MK, Brophy GM, et al. Consensus summary statement of the international multidisciplinary consensus conference on multimodality monitoring in neurocritical care : a statement for healthcare professionals from the Neurocritical Care Society and the European Society of Intensive Care Medicine. Intensive Care Med. 2014:40:1189-209.

4. Czosnyka M, Miller C. Participants in the international multidisciplinary consensus conference on multimodality monitoring. Monitoring of cerebral autoregulation. Neurocrit Care. 2014;21 (Suppl 2):95-102

5. Carney N, Totten AM, O'Reilly C, Ullman JS, Hawryluk GWJ, Bell MJ, et al. Guidelines for the management of severe traumatic brain injury. Fourth Edn Neurosurg. 2017;80:6-15.

6. Chesnut R, Aguilera S, Buki A, Bulger E, Citerio G, Cooper DJ, et al. A management algorithm for adult patients with both brain oxygen and intracranial pressure monitoring: the Seattle International Severe Traumatic Brain Injury Consensus Conference (SIBICC). Intensive Care Med. 2020;46(5):919-29. https://doi.org/10.1007/s00134-019-05900-x.

7. Hawryluk GWJ, Aguilera S, Buki A, Bulger E, Citerio G, Cooper DJ, et al. A management algorithm for patients with intracranial pressure monitoring: the Seattle International Severe Traumatic Brain Injury Consensus Conference (SIBICC). Intensive Care Med. 2019;45:1783-94.
8. Okonkwo DO, Shutter LA, Moore C, Temkin NR, Puccio AM, Madden CJ, et al. Brain oxygen optimization in severe traumatic brain injury phase-ll: a phase II randomized trial. Crit Care Med. 2017:45:1907-14.

9. Beqiri E, Smielewski P, Robba C, Czosnyka M, Cabeleira MT, Tas J, et al. Feasibility of individualised severe traumatic brain injury management using an automated assessment of optimal cerebral perfusion pressure: the COGiTATE phase II study protocol. BMJ Open. 2019;9:e030727.

10. Czosnyka M, Smielewski P, Kirkpatrick P, Laing RJ, Menon D, Pickard JD. Continuous assessment of the cerebral vasomotor reactivity in head injury. Neurosurgery. 1997;41:11-7; (discussion 17-19).

11. Zeiler FA, Ercole A, Czosnyka M, Smielewski P, Hawryluk G, Hutchinson PJA, et al. Continuous cerebrovascular reactivity monitoring in moderate/ severe traumatic brain injury: a narrative review of advances in neurocritical care. Br J Anaesth. 2020. https://doi.org/10.1016/j.bja.2019.11.031.

12. Calviello L, Donnelly J, Cardim D, Robba C, Zeiler FA, Smielewski P, et al. Compensatory-reserve-weighted intracranial pressure and its association with outcome after traumatic brain injury. Neurocrit Care. 2018;28:212-20.

13. Zeiler FA, Ercole A, Cabeleira M, Beqiri E, Zoerle T, Carbonara M, et al. Compensatory-reserve-weighted intracranial pressure versus intracranial pressure for outcome association in adult traumatic brain injury: a CENTER-TBI validation study. Acta Neurochir (Wien). 2019;161:1275-84.

14. Gao L, Smielewski P, Czosnyka M, Ercole A. Cerebrovascular signal complexity six hours after intensive care unit admission correlates with outcome after severe traumatic brain injury. J Neurotrauma. 2016;33:2011-8.

15. Zeiler FA, Ercole A, Placek MM, Hutchinson PJ, Stocchetti N, Czosnyka M, et al. Association between physiological signal complexity and outcomes in moderate and severe traumatic brain injury: a CENTER-TBI exploratory analysis of multi-scale entropy. J Neurotrauma. 2020. https://doi. org/10.1089/neu.2020.7249.

16. Lavinio A, Ene-lordache B, Nodari I, Girardini A, Cagnazzi E, Rasulo F, et al. Cerebrovascular reactivity and autonomic drive following traumatic brain injury. Acta Neurochir Suppl. 2008;102:3-7.

17. Hasen M, Almojuela A, Zeiler FA. Autonomic dysfunction and associations with functional and neurophysiological outcome in moderate/ severe traumatic brain injury: a scoping review. J Neurotrauma. 2019;36:1491-504.

18. Zeiler FA, Donnelly J, Calviello L, Smielewski P, Menon DK, Czosnyka M. Pressure autoregulation measurement techniques in adult traumatic brain injury, part Il: a scoping review of continuous methods. J Neurotrauma. 2017;34:3224-37.

19. Bennis FC, Teeuwen B, Zeiler FA, Elting JW, van der Naalt J, Bonizzi P, et al. Improving prediction of favourable outcome after 6 months in patients with severe traumatic brain injury using physiological cerebral parameters in a multivariable logistic regression model. Neurocrit Care. 2020;33(2):542-51. https://doi.org/10.1007/s12028-020-00930-6.

20. Sorrentino E, Diedler J, Kasprowicz M, Budohoski KP, Haubrich C, Smielewski $P$, et al. Critical thresholds for cerebrovascular reactivity after traumatic brain injury. Neurocrit Care. 2012;16:258-66.

21. Donnelly J, Czosnyka M, Adams H, Cardim D, Kolias AG, Zeiler FA, et al. Twenty-five years of intracranial pressure monitoring after severe traumatic brain injury: a retrospective. Single-Center Anal Neurosurg. 2019;85:E75-82

22. Zeiler FA, Ercole A, Beqiri E, Cabeleira M, Thelin EP, Stocchetti N, et al. Association between cerebrovascular reactivity monitoring and mortality is preserved when adjusting for baseline admission characteristics in adult TBI: a CENTER-TBI study. J Neurotrauma. 2020;37(10):1233-41. https://doi. org/10.1089/neu.2019.6808.

23. Zeiler FA, Ercole A, Cabeleira M, Zoerle T, Stocchetti N, Menon DK, et al. Univariate comparison of performance of different cerebrovascular reactivity indices for outcome association in adult TBI: a CENTER-TBI study. Acta Neurochir (Wien). 2019;161:1217-27.

24. Brady KM, Lee JK, Kibler KK, Easley RB, Koehler RC, Shaffner DH. Continuous measurement of autoregulation by spontaneous fluctuations in cerebral perfusion pressure: comparison of 3 methods. Stroke. 2008;39:2531-7.

25. Zeiler FA, Lee JK, Smielewski P, Czosnyka M, Brady K. Validation of intracranial pressure-derived cerebrovascular reactivity indices against the lower limit of autoregulation, Part II: experimental model of arterial hypotension. J Neurotrauma. 2018;35:2812-9. 
26. Zeiler FA, Donnelly J, Calviello L, Lee JK, Smielewski P, Brady K, et al. Validation of pressure reactivity and pulse amplitude indices against the lower limit of autoregulation, part I: experimental intracranial hypertension. J Neurotrauma. 2018;35:2803-11.

27. Zeiler FA, Donnelly J, Smielewski P, Menon DK, Hutchinson PJ, Czosnyka M. Critical thresholds of intracranial pressure-derived continuous cerebrovascular reactivity indices for outcome prediction in noncraniectomized patients with traumatic brain injury. J Neurotrauma. 2018;35:1107-15.

28. Zeiler FA, Beqiri E, Cabeleira M, Hutchinson PJ, Stocchetti N, Menon DK, et al. Brain tissue oxygen and cerebrovascular reactivity in traumatic brain injury: a CENTER-TBI exploratory analysis of insult burden. J Neurotrauma. 2020;37(17):1854-63. https://doi.org/10.1089/neu.2020.7024.

29. Zeiler FA, Ercole A, Beqiri E, Cabeleira M, Aries M, Zoerle T, et al. Cerebrovascular reactivity is not associated with therapeutic intensity in adult traumatic brain injury: a CENTER-TBI analysis. Acta Neurochir (Wien). 2019;161(9):1955-64. https://doi.org/10.1007/s00701-019-03980-8.

30. Froese L, Batson C, Gomez A, Dian J, Zeiler FA. The limited impact of current therapeutic interventions on cerebrovascular reactivity in traumatic brain injury: a narrative overview. Neurocrit Care. 2020. https://doi. org/10.1007/s12028-020-01003-4

31. Steiner LA, Czosnyka M, Piechnik SK, Smielewski P, Chatfield D, Menon DK, et al. Continuous monitoring of cerebrovascular pressure reactivity allows determination of optimal cerebral perfusion pressure in patients with traumatic brain injury. Crit Care Med. 2002;30:733-8.

32. Aries MJH, Czosnyka M, Budohoski KP, Steiner LA, Lavinio A, Kolias AG, et al. Continuous determination of optimal cerebral perfusion pressure in traumatic brain injury. Crit Care Med. 2012;40:2456-63.

33. Needham E, McFadyen C, Newcombe V, Synnot AJ, Czosnyka M, Menon D. Cerebral perfusion pressure targets individualized to pressure-reactivity index in moderate to severe traumatic brain injury: a systematic review. J Neurotrauma. 2017:34:963-70.

34. Güiza F, Depreitere B, Piper I, Citerio G, Chambers I, Jones PA, et al. Visualizing the pressure and time burden of intracranial hypertension in adult and paediatric traumatic brain injury. Intensive Care Med. 2015:41:1067-76.

35. Güiza F, Meyfroidt G, Piper I, Citerio G, Chambers I, Enblad P, et al. Cerebral perfusion pressure insults and associations with outcome in adult traumatic brain injury. J Neurotrauma. 2017;34:2425-31.
36. Donnelly J, Güiza F, Depreitere B, Meyfroidt G, Czosnyka M, Smielewski P. Visualising the pressure-time burden of elevated intracranial pressure after severe traumatic brain injury: a retrospective confirmatory study. $\mathrm{Br}$ J Anaesth. 2020. https://doi.org/10.1016/j.bja.2020.09.018.

37. Budohoski KP, Czosnyka M, Kirkpatrick PJ, Smielewski P, Steiner LA, Pickard JD. Clinical relevance of cerebral autoregulation following subarachnoid haemorrhage. Nat Rev Neurol. 2013;9:152-63.

38. Budohoski KP, Czosnyka M, Smielewski P, Kasprowicz M, Helmy A, Bulters $D$, et al. Impairment of cerebral autoregulation predicts delayed cerebral ischemia after subarachnoid hemorrhage: a prospective observational study. Stroke. 2012;43:3230-7.

39. Bijlenga P, Czosnyka M, Budohoski KP, Soehle M, Pickard JD, Kirkpatrick PJ, et al. "Optimal cerebral perfusion pressure" in poor grade patients after subarachnoid hemorrhage. Neurocrit Care. 2010;13:17-23.

40. Budohoski KP, Czosnyka M, Smielewski P, Varsos GV, Kasprowicz M, Brady KM, et al. Cerebral autoregulation after subarachnoid hemorrhage: comparison of three methods. J Cereb Blood Flow Metab Off J Int Soc Cereb Blood Flow Metab. 2013;33:449-56.

41. Soehle M, Czosnyka M, Pickard JD, Kirkpatrick PJ. Continuous assessment of cerebral autoregulation in subarachnoid hemorrhage. Anesth Analg. 2004;98:1133-9; (table of contents).

42. Svedung Wettervik T, Howells T, Lewén A, Ronne-Engström E, Enblad P. Temporal dynamics of ICP, CPP, PRx, and CPPopt in high-grade aneurysmal subarachnoid hemorrhage and the relation to clinical outcome. Neurocrit Care. 2020. https://doi.org/10.1007/s12028-020-01162-4.

43. Zeiler FA, Donnelly J, Menon DK, Smielewski P, Hutchinson PJA, Czosnyka $M$. A description of a new continuous physiological index in traumatic brain injury using the correlation between pulse amplitude of intracranial pressure and cerebral perfusion pressure. J Neurotrauma. 2018;35(7):96374. https://doi.org/10.1089/neu.2017.5241.

44. Aries MJH, Czosnyka M, Budohoski KP, Kolias AG, Radolovich DK, Lavinio A, et al. Continuous monitoring of cerebrovascular reactivity using pulse waveform of intracranial pressure. Neurocrit Care. 2012;17:67-76. 